\title{
Adherencia al uso de audífonos en adultos mayores chilenos con hipoacusia
}

\section{Adherence to hearing aids use in Chilean older adults with hearing loss}

\author{
Felipe Cardemil M. ${ }^{1,2}$, Tamara Barría ${ }^{1}$, Eduardo Fuentes-López ${ }^{3}$, Daniel Muñoz S. ${ }^{1}$, \\ Lorena Aguayo G. ${ }^{1}$, Adrián Fuente C. ${ }^{4}$, Maritza Rahal E. ${ }^{1,5}$
}

\section{Resumen}

Introducción: Se estima que hasta un 30\% de la población mayor de 65 años presenta hipoacusia. Una intervención validada para la hipoacusia en adultos mayores es el uso de audífonos, pero el problema es que hasta un 50\% de los adultos mayores no los utiliza. Objetivo: Estimar la adherencia al uso de audífonos en adultos mayores beneficiarios del programa GES de "Hipoacusia bilateral en mayores de 65 años que requieran audífonos” en un hospital en Santiago, Chile. Material y Método: Se utilizó la rama control de un ensayo clínico aleatorizado para estimar la adherencia al uso de audífono con cuestionarios validados y estructurados, por encuestadores entrenados que acudieron a los tres, seis, nueve y doce meses desde la intervención al domicilio de los beneficiarios del programa. Resultados: Se incluyeron 101 adultos mayores. El promedio de edad fue 76,3 años. Al analizar la adherencia considerando un criterio de uso del audífono mayor a $8 \mathrm{~h}$ al día, cada día, durante las últimas dos semanas, la adherencia a 3 meses fue un 56,8\%, a 6 meses un $62,6 \%$, a 9 meses un $63,2 \%$ y a 12 meses un 54,5\%. Al analizar considerando uso entre 4-8 h/diarias, la adherencia del período fue entre $73,8 \%$ y $87,3 \%$. Según el cuestionario del Dr. Yueh, la adherencia a 3 meses fue un $64,7 \%$, a 6 meses un $63,9 \%$, a 9 meses un $73,7 \%$ y a 12 meses un $67,8 \%$. Conclusión: Los resultados del presente análisis muestran una excelente adherencia al uso del audífono en Chile, lo que representa una cifra positiva respecto a la efectividad de la política pública.

Palabras clave: hipoacusia, audífonos, adherencia.

\begin{abstract}
Introduction: It is estimated that up to $30 \%$ of the population over 65 years of age present hearing loss. A validated intervention for hearing loss in older adults is the use of hearing aids, but the problem is that up to 50\% of older adults do not use them. Aim: To estimate the hearing aid adherence in elderly population with hearing loss who are beneficiaries of the GES program "Bilateral hearing loss in people over 65 who require hearing aids" in a hospital in Santiago, Chile. Material and Method: The control arm of a randomized clinical trial was used to estimate adherence to hearing aid with validated and structured questionnaires, by trained interviewers who visited the beneficiaries of the program at three, six, nine and twelve months posterior to the intervention. Results: 101 older adults were included. The average age was 76.3 years. When analyzing adherence as "use for more than 8 hours a day during the last two weeks", adherence at 3 months was $56.8 \%$, at 6 months $62.6 \%$, at 9 months $63.2 \%$, and at 12 months $54.5 \%$. When analyzing considering use between 4-8 h/day, the adherence during the follow-up was between $73.8 \%$ and $87.3 \%$. According to Dr. Yueh's questionnaire, adherence at 3 months was $64.7 \%$, at 6 months $63.9 \%$, at 9 months $73.7 \%$, and at 12 months $67.8 \%$. Conclusion: The results of the present analysis show an excellent adherence to hearing aids in a group of Chilean population, which represents a positive outcome regarding the effectiveness of this particular public policy.
\end{abstract}

Keywords: Hearing loss, hearing aids, adherence.
${ }^{1}$ Departamento de Otorrinolaringología, Facultad de Medicina, Universidad de Chile. Santiago, Chile. 2Departamento de Otorrinolaringología, Clínica Las Condes. Santiago, Chile. ${ }^{3}$ Carrera de Fonoaudiología, Departamento de Ciencias de la Salud, Facultad de Medicina, Pontificia Universidad Católica de Chile. Santiago, Chile. ${ }^{4}$ Departamento de Fonoaudiología, Facultad de Medicina, Universidad de Chile. Santiago, Chile. ${ }^{5}$ Servicio de Otorrinolaringología, Hospital Barros Luco Trudeau, Facultad de Medicina, Universidad de Chile. Santiago, Chile.

Estudio financiado con aporte de proyectos FONIS SA1112199 y FONDECYT 1151502. Registro NCT02264314 en www. clinicaltrials.gov

Recibido el 22 de mayo de 2020. Aceptado el $1 \mathrm{de}$ agosto de 2020 .

Correspondencia: Felipe Cardemil M. Departamento de Otorrinolaringología, Facultad de Medicina Universidad de Chile. Santiago, Chile. Email: felipecardemil@med. uchile.cl 


\section{Introducción}

El envejecimiento progresivo de la población mundial es una preocupación reconocida por la mayoría de las propuestas de políticas públicas en diversas áreas, incluida salud. Se estima que cerca del $23 \%$ de las personas de países desarrollados y el $9 \%$ de las personas de países en vías de desarrollo tienen 60 años o más, cifras que tienden al alza en diferentes proyecciones que estiman que para el año 2100 hasta un $34 \%$ y un $27 \%$ de la población se encontrará en ese grupo etáreo en esos países, respectivamente ${ }^{1}$. El porcentaje de crecimiento en el número de personas de 65 años o más aumentó entre un $18 \%$ y un $50 \%$ entre el período 2010-2020 en todas las regiones del mundo ${ }^{2}$.

Considerada tradicionalmente como una condición natural del envejecimiento, la hipoacusia es una condición más frecuente a medida que aumenta la edad ${ }^{3}$. La prevalencia de hipoacusia ha sido a menudo subestimada debido a la lenta progresión de esta condición. En tal contexto, la hipoacusia resulta una condición de salud subdiagnosticada y subtratada ${ }^{4,5}$. La estimación del $15 \%$ de la población mundial adulta con algún grado de hipoacusia se eleva a poco más del $30 \%$ cuando sólo se considera la población mayor de 65 años $^{6}$ y en un $60 \%$ en mayores de 85 años $^{7}$. Esto da cuenta que el número de personas con pérdida de audición podrá aumentar en la medida que se mantenga la tendencia de los cambios demográficos ${ }^{6}$. Las cifras referentes a la realidad chilena sostienen que el $91,1 \%$ de los mayores de 80 años presentaban algún grado de hipoacusia de acuerdo con la Encuesta Nacional de Salud del año $2003^{8}$. La versión de esta encuesta realizada el año 2009-10 estimó que un 52,4\% de las personas de 65 años o más presentaban esta condición en algún grado 9

Las consecuencias de la pérdida de capacidad auditiva en el adulto mayor se manifiestan en problemas de la comprensión del habla, especialmente cuando existe ruido de fondo o reverberación ${ }^{10,11}$. Los problemas de comunicación oral se asocian con una reducción en la calidad de vida, tanto en quien padece esta dificultad como en su entorno ${ }^{12,13}$. Se ha descrito la asociación de hipoacusia con cuadros de deterioro cognitivo, siendo un factor de riesgo conocido para algunos tipo de demencia ${ }^{14} \mathrm{y}$ trastornos de la salud mental como ansiedad y depresión ${ }^{15-18}$. Incluso se ha señalado un mayor riesgo de mortalidad asociado a problemas auditivos ${ }^{19}$.

Las ayudas auditivas externas como los audífonos resultan ser la opción de tratamiento de mayor disponibilidad para quienes sufren de pérdida auditiva relacionada al envejecimiento $^{20}$. El uso de estos dispositivos ha aumentado exponencialmente, estimando que en EE. UU. su utilización se ha incrementado en casi un 300\% durante el decenio comprendido entre 1996 y $2006^{21}$. Sin embargo, aún existen desafíos en su acceso, considerando que anualmente sólo se produce el $10 \%$ de la necesidad global en audífonos. El uso de audífonos en las personas adultas mayores se fundamenta en los positivos resultados que tendrían en relación con menor ansiedad y depresión ${ }^{22-24}$, incluso comparado con la población geriátrica general ${ }^{25}$; el mejoramiento de niveles audiológicos globales y específicos de calidad de vida en personas con hipoacusia ${ }^{26} y$ la disminución de consecuencias psicológicas, sociales y emocionales de la hipoacusia luego de la implementación con audífonos ${ }^{27}$.

En este sentido, uno de los principales problemas descritos es la adherencia al uso del audífono. Se describe que entre el 30\% y 45\% de los adultos mayores que reciben audífonos por primera vez no los utilizan, cifra que puede llegar hasta el 50\% de no uso en seguimientos a distintos períodos de tiempo ${ }^{28,29}$. La explicación a esta subutilización se centra en la aceptación de la condición de salud (y, por ende, el uso del dispositivo) y en la adaptación a la prótesis auditiva, proceso de muchas dificultades y prejuicios que se relacionan con aspectos tanto técnicos, estéticos y sociales ${ }^{30}$. Uno de los problemas de una potencial baja adherencia al uso de una intervención es que los encargados de la toma de decisiones de los programas de salud pueden considerar que un programa es poco eficiente y efectivo si las personas no adhieren a la intervención, lo que podría decantar en una contracción o una suspensión del programa de salud.

\section{Objetivo}

El objetivo del presente estudio fue estimar la adherencia al uso de audífono en adultos 
mayores en Chile beneficiarios del programa GES de "Hipoacusia bilateral en mayores de 65 años que requieran audífonos", mediante la utilización de un estudio prospectivo y con evaluación seriada en el domicilio, a través de una encuesta periódica estructurada.

\section{Material y Método}

Se incluyeron los participantes pertenecientes a la rama control de un ensayo clínico aleatorizado (protocolo NCT02264314, disponible en www.clinicaltrials.gov). En este se aleatorizaron 202 participantes adultos mayores usuarios de audífonos beneficiarios del programa GES "Hipoacusia bilateral en mayores de 65 años que requieran audífonos" que acudieron a recibir atención en salud en el Servicio de Otorrinolaringología del Hospital Barros Luco Trudeau en dos grupos: una rama activa que consistía en una intervención a través de un programa teleeducativo para mejorar adherencia y calidad de vida y una rama control en la que no se realizaba la intervención; ambos grupos fueron seguidos durante 12 meses posterior a la entrega de audífonos. Para los fines del presente estudio, se consideró solamente a los pacientes que ingresaron de manera prospectiva y fueron asignados a la rama control, es decir, que no recibieron la intervención, para poder estimar la adherencia basal al uso del audífono en población habitual. El desenlace principal a evaluar fue adherencia al uso del audífono. El estudio se realizó desde junio del año 2012 hasta diciembre del año 2014. El protocolo del estudio contó con la aprobación del Comité de Ética de Investigación en Seres Humanos de la Facultad de Medicina de la Universidad de Chile y cada participante firmó un consentimiento informado para los fines de este estudio.

Como criterio de inclusión se consideraron participantes que tuvieran hipoacusia bilateral en rango moderado igual o mayor a 40 decibeles, edad igual o mayor a 65 años al momento del enrolamiento, contar con familiar o acompañante de soporte y firmar consentimiento informado para la participación en el proyecto. Los criterios de exclusión fueron deterioro cognitivo que afectara la funcionalidad del adulto mayor o demencia medida a través del puntaje de la prueba Mini-Mental Test de Folstein abreviada, incapacidad física o mental para entender los cuestionarios del estudio o la presencia de patología estructural del oído que genera la hipoacusia (como otitis media crónica, tumor del conducto auditivo externo, tumor de ángulo pontocerebeloso u otra).

Se consideraron las variables edad, sexo, promedio tonal puro entre las frecuencias 500, 1000, 2000 y 4000 Hz (PTP) aéreo y óseo, discriminación de la palabra, tipo de hipoacusia (conductiva, sensorioneural, mixta) y forma de la curva auditiva, escolaridad, uso previo de audífono, marca y modelo de audífono recibido, comorbilidades y predisposición al uso de audífono.

Para evaluar adherencia se utilizó la pregunta inicial del "International Outcome Inventory for Hearing Aids" (IOI-HA, conocido en español como Cuestionario Internacional de Resultados del Uso de Audífono, CIRUA), que evalúa específicamente adherencia con una de sus preguntas. Además, se evaluó adherencia mediante el cuestionario que utiliza el Dr. Bevan Yueh de la Universidad de Minnesota, que indaga específicamente adherencia al uso del audífono (Figura 1). Para hacer más operativo el concepto de "adherencia" y considerando la pregunta del CIRUA, se definió como criterio previo al inicio del estudio, un uso del audífono de al menos 8 horas al día en cada una de las evaluaciones, considerando las últimas dos semanas previas. A su vez, considerando los

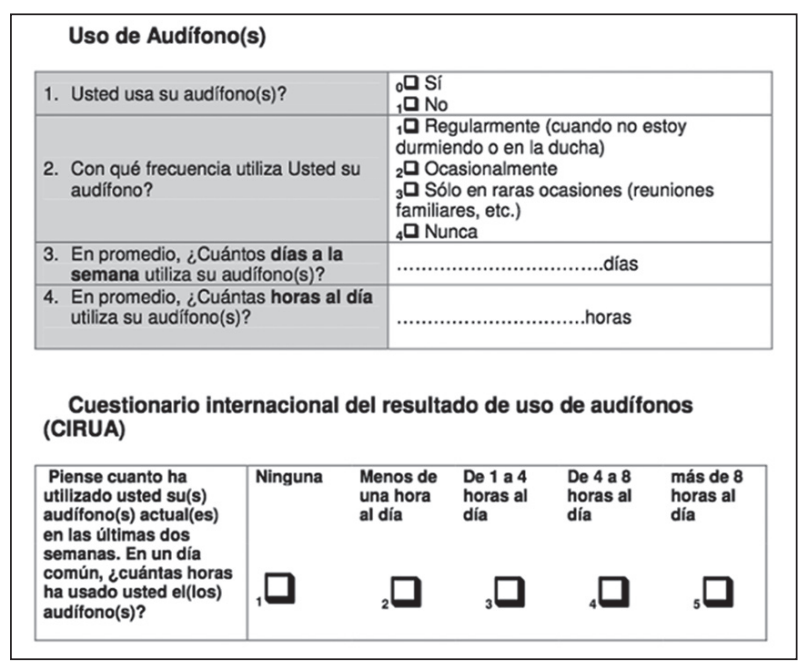

Figura 1. Cuestionario del Dr. Bevan Yueh y cuestionarios CIRUA para evaluar adherencia en usuarios de audífonos. 
Tabla 1. Adherencia según CIRUA y cuestionario del Dr. Bevan Yueh a audífono en pacientes con presbiacusia en Chile que se acogen al régimen GES

\begin{tabular}{ccc}
\hline $\begin{array}{c}\text { Adherencia } \\
\text { (uso mayor a } \mathbf{8} \text { h/día) }\end{array}$ & $\begin{array}{c}\text { \% Grupo Control según } \\
\text { CIRUA }\end{array}$ & $\begin{array}{c}\text { \% Grupo Control según cuestionario } \\
\text { Yueh }\end{array}$ \\
\hline 3 meses & $56,82 \%$ & $64,77 \%$ \\
6 meses & $62,65 \%$ & $63,95 \%$ \\
9 meses & $63,29 \%$ & $73,75 \%$ \\
\hline 12 meses & $54,55 \%$ & $67,82 \%$ \\
\hline En 2 o más evaluaciones & $76,19 \%$ & $85,37 \%$ \\
\hline
\end{tabular}

tiempos consultados en el cuestionario del Dr. Yueh, se evaluó la consistencia de la adherencia con mayor exigencia, integrándola como un uso al menos de $8 \mathrm{~h}$ al día con una regularidad de 4 o más días a la semana.

Todos los participantes fueron evaluados en su domicilio a los 3, 6, 9 y 12 meses desde la intervención para indagar respecto a la adherencia al uso del audífono. Las evaluaciones se llevaron a cabo por encuestadores entrenados enmascarados respecto a la asignación aleatoria a la intervención. Los encuestadores que evaluaron en el domicilio a los participantes fueron entrenados en cuatro sesiones por uno de los investigadores con experiencia en aplicación de cuestionarios.

Para el análisis estadístico se utilizó estadística descriptiva, con promedio y desviación estándar (DE), así como frecuencia absoluta y relativa. Los datos fueron analizados con Stata 12.

\section{Resultados}

Al finalizar el período de reclutamiento, 101 participantes se encontraban asignados a la rama control, por lo tanto, conformaron la muestra que se utilizó para evaluar adherencia. Cada participante fue evaluado cuatro veces en su domicilio y las pérdidas de seguimiento fueron menores al 20\%. El promedio (DE) de edad en la muestra fue de 76,3 años $( \pm 6,9)$, con un $56,4 \%$ de la muestra siendo de sexo femenino. Un 38,4\% de la muestra presentó escolaridad básica incompleta, un 11,1\% básica completa, un $19,2 \%$ enseñanza media incompleta, un $20,2 \%$ media completa y un $11,1 \%$ educación superior. Un $70 \%$ de la muestra presentaba hipertensión arterial, un 25\% diabetes mellitus y un $13 \%$ dislipidemia.

Respecto a variables auditivas, el promedio de PTP óseo fue de 49,7 (10,01) dB HL, mientras que el PTP aéreo fue de 57,03 $(14,8)$ dB HL. El promedio de discriminación de ambos oídos para palabras monosilábicas fue de $72,3 \%(24,5 \%)$. Un $85,9 \%$ de la muestra presentó una hipoacusia de tipo sensorioneural, correspondiendo a un $72,2 \%$ una curva descendente. Un $27,9 \%$ de la muestra había usado audífonos anteriormente. Un 94,9\% de la muestra tenía una predisposición "buena" o "muy buena" al uso del audífono, mientras que sólo un 5,1\% refería una predisposición "regular", "mala" o "muy mala" al uso de éste.

$\mathrm{Al}$ analizar la adherencia como uso mayor a ocho horas al día durante las últimas dos semanas, según CIRUA, la adherencia a 3 meses fue un $56,8 \%$, a 6 meses un $62,6 \%$, a 9 meses un $63,2 \%$ y a 12 meses un $54,5 \%$. Según el cuestionario del Dr. Yueh, la adherencia a 3 meses fue un $64,7 \%$, a 6 meses un 63,9\%, a 9 meses un $73,7 \%$ y a 12 meses un $67,8 \%$. El desenlace compuesto de dos de las cuatro mediciones o más y el detalle por evaluación se describe en la Tabla 1 y la Figura 2. Al evaluar adherencia con CIRUA como uso entre 4 y $8 \mathrm{~h}$ diarias durante las últimas 2 semanas, la adherencia a 3, 6, 9, 12 meses y el desenlace combinado de 2 evaluaciones o más fue de $73,8 \%, 83,1 \%$, $87,3 \%, 84 \%$ y $94,2 \%$ respectivamente.

$\mathrm{Al}$ analizar la adherencia como uso mayor a 8 h por día en 4 días a la semana o más según el cuestionario del Dr. Yueh, la adherencia a 3 meses fue un $60,2 \%$, a 6 meses un $59,3 \%$, a 9 meses un $70 \%$ y a 12 meses un $66,6 \%$. Por úl- 


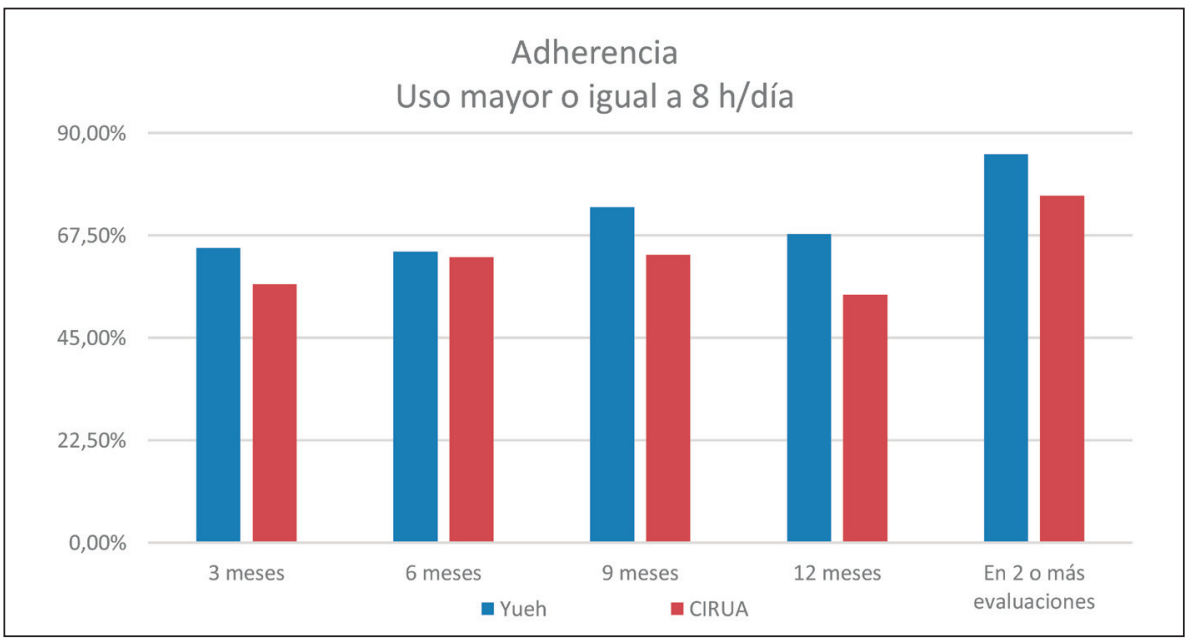

Figura 2. Adherencia según CIRUA y cuestionario del Dr. Bevan Yueh a audífono en pacientes con presbiacusia en Chile que se acogen al régimen GES. timo, al evaluar la adherencia como frecuencia de uso regularmente, la adherencia a 3, 6, 9, 12 meses y el desenlace combinado de 2 evaluaciones o más fue de $69,3 \%, 73,2 \%, 81,2 \%, 80 \%$ y $90,3 \%$ respectivamente.

\section{Discusión}

En políticas públicas o programas de salud, es muy importante medir resultados. Con el objetivo de ofrecer una atención de salud que garantice oportunidad, calidad y protección financiera para problemas de salud con alto impacto sanitario y así, disminuir la brecha de oportunidad entre los subsistemas de salud público y privado, la última reforma de salud es considerada el mayor esfuerzo del sector en los últimos 25 años31. Diseñada desde la mirada del cumplimiento de objetivos sanitarios, esta reforma tiene en la Ley AUGE -ahora llamada Régimen de Garantías Explícitas en Salud (GES) - el principal constituyente de este esfuerzo. En este sentido, la evaluación de adherencia al uso de audífono es un desenlace importante relativo a la garantía GES de "Hipoacusia bilateral en personas de 65 años y más que requieren uso de audífono”, la que fue uno de los 56 problemas incorporados al inicio del GES. Enfocado en personas de 65 años y más, su abordaje implicaba la entrega de audífonos a todo quien cumpla con los criterios diagnósticos ${ }^{15}$.
En Chile, se ha sugerido que un 53\% de las personas a las que se les prescribió el uso de audífonos lo utilizan ${ }^{8}$, cifra con una amplia variabilidad de acuerdo al tipo de mediciones y usuarios, oscilando entre un $40 \%$ a un $80 \%{ }^{32-35}$. Tales cifras dan cuenta de la importancia de determinar los motivos por los cuales las personas no usan los audífonos. Incluso, se ha propuesto en la literatura que en países donde existe acceso a dispositivos auditivos, como Chile, es imperioso determinar por qué la gente no usa el audífono, de manera tal de intervenir en estos factores ${ }^{36}$.

Los estudios publicados previamente con resultados de adherencia al audífono en Chile entregan resultados interesantes, pero disímiles. Por ejemplo, en un estudio se contactó telefónicamente a 179 adultos mayores de 65 años que recibieron audífonos por el Programa del Adulto Mayor de dos hospitales de Santiago ${ }^{32}$. Se les preguntó directamente a los beneficiarios o a un familiar que viviera con él si usaban o no su audífono, sin especificar el criterio con el que se consideraría el uso, estableciéndose que un $79,2 \%$ se encontraba usándolo a un año de seguimiento. En otro estudio se visitó a 42 adultos mayores del Servicio de Salud Aconcagua para indagar respecto a adherencia al uso del audífono ${ }^{33}$, la que fue definida como personas que usaban su audífono a diario y se encontraban usándolo durante la visita. Los autores reportan una adherencia del 90\% considerando los parámetros de la Encuesta 
Nacional de Salud, la que disminuía a 40\% si se consideraban los criterios que los autores proponían y a un $21 \%$ de uso correcto del audífono. En otro estudio, 84 pacientes atendidos en un centro de salud privado de Santiago fueron contactados telefónicamente ${ }^{34}$, estableciéndose que sólo un 76,2\% de éstos tuvo acceso finalmente al audífono, y que un $48 \%$ de los beneficiarios utilizaba el dispositivo uno o más días a la semana ${ }^{34}$. En otro estudio se contactó telefónicamente a 32 adultos mayores que no concurrieron al tercer control posterior a la entrega del audífono en el Hospital Carlos Van Buren de Valparaíso ${ }^{35}$, describiéndose que un $59,4 \%$ de estos ocupaba siempre su audífono. Respecto a los resultados de estos estudios llama la atención la variabilidad de los resultados de adherencia, la que puede ser atribuida a las diferentes metodologías utilizadas, así como a diferentes criterios para definir "adherencia"; en cualquier caso, el problema de la adherencia terapéutica debiera ser evaluado siempre mediante metodologías adecuadas, ya que lo que se busca es definir el valor más exacto a la realidad, para objetivar la efectividad de una intervención (en este caso, un programa universal de entrega de audífonos). En segundo lugar, llama la atención que casi un cuarto de los pacientes atendidos en el estudio del centro de salud privado no haya obtenido finalmente el audífono, a pesar de tener la indicación de usarlo según los criterios GES. Por último, hay que ser cuidadoso en el momento que se utiliza una metodología cuantitativa, como la del presente estudio y la de los otros estudios nacionales, para indagar y analizar respecto a motivos o causas, en este caso de no uso de audífonos. La metodología adecuada para evaluar las razones por las cuales un grupo de personas hace o deja de hacer algo (en este caso, usar el audífono) debe ser idealmente de tipo cualitativo ${ }^{37}$, a través de grupos focales o entrevistas individuales, por ejemplo, siendo un desafío a abordar en futuras investigaciones.

La estimación de adherencia del presente estudio, de entre un $54,5 \%$ a un $73,7 \%$ con un criterio estricto luego de 12 meses de uso, es una cifra muy alentadora. En primer lugar, porque la metodología utilizada a través de visitas domiciliarias seriadas con encuestadores entrenados a 101 participantes representa un esfuerzo serio por estimar fehacientemente la adherencia al uso del audífono en adultos mayores chilenos beneficiarios del programa GES. En segundo lugar, porque se utilizaron criterios muy estrictos para definir adherencia, de al menos $8 \mathrm{~h}$ diarias. $\mathrm{Al}$ analizar los resultados considerando criterios menos estrictos (entre 4 y $8 \mathrm{~h}$ diarias) la adherencia es aún mayor, entre $73,8 \%$ y $87,3 \%$, lo que representa una excelente proporción de uso del audífono si consideramos que muchas personas lo usan para actividades puntuales, como durante el almuerzo, reuniones familiares, asistencia a reuniones religiosas o actividades sociales. Incluso si se considera la pregunta del cuestionario del Dr. Yueh "frecuencia de uso del audífono regularmente" (pregunta 2 del cuestionario, Figura 1), la adherencia fue entre $69,3 \%$ y $81,2 \%$, lo que nuevamente representa un excelente resultado. Por último, para evaluar adherencia se utilizaron dos instrumentos: la escala CIRUA, que es el único instrumento validado que incluye una pregunta específica sobre adherencia y el instrumento de un reconocido autor internacional; el utilizar estos dos instrumentos se definió luego de un proceso de análisis de las alternativas existentes y las ventajas y desventajas de utilizar estos cuestionarios o realizar preguntas abiertas no validadas.

Como se mencionó previamente, la evaluación de resultados en políticas públicas es muy relevante, debido a que es la única manera que tienen los tomadores de decisión ("policymakers") para saber si los programas de salud son efectivos o no ${ }^{38}$. En este sentido, es muy importante evaluar los desenlaces de los programas con metodologías adecuadas, porque las publicaciones científicas son utilizadas con frecuencia por las instituciones así como por los tomadores de decisión para definir materias respecto a la continuidad o al aumento de los programas ${ }^{39,40}$. Por este motivo, al difundir cifras que pueden subestimar la realidad, es posible comprometer algunos beneficios o programas, que desde el punto de vista médico existe evidencia de su efectividad, como es la entrega de audífonos para adultos mayores con hipoacusia. El resultado de adherencia del presente estudio, en torno al $60 \%$, es muy positivo, porque sugiere que la utilización del audífono es superior a lo que se pensaba previamente, lo que da luces respecto al beneficio real del programa de salud $y$ 
permite sostener con cifras concretas que éste debe mantenerse y eventualmente mejorarse, con implementación biaural o más temprana edad del audífono, como ha sido sugerido en otras publicaciones ${ }^{41}$.

En relación a debilidades de este estudio, siempre existe una posible fuente de sesgo cuando las personas saben que son participantes en un estudio y se evalúan con preguntas de cuestionarios, ya que puede haber una sobreestimación en relación a la respuesta que ellos dan. A pesar de que este estudio intentó controlar todas las posibles fuentes de confusión con evaluaciones en domicilio con cuestionarios estructurados por encuestadores entrenados, los participantes del estudio estaban al tanto de su enrolamiento en éste, por lo que hay que considerar eso al momento de ponderar la estimación reportada en este estudio. Además, es necesario considerar que la adherencia al uso del audífono es un desenlace intermedio, que en teoría es un reflejo de otros aspectos en el sentido que si una persona con hipoacusia usa adecuadamente el audífono se podría esperar que se integrara socialmente mejor, que tuviera mejor calidad de vida e incluso tener mejores indicadores de salud mental, entre otros, pero la adherencia al audífono en sí misma no asegura que estos otros desenlaces van a ocurrir ${ }^{4,14}$.

Finalmente, es importante plantearse las razones del por qué algunos adultos mayores que han recibido un audífono dejan de utilizarlo al año de su entrega. Futuras investigaciones debieran enfocarse en determinar qué factores se relacionan al fracaso de la adaptación de audífonos en el $45 \%$ a $33 \%$ de los adultos mayores que no usan el audífono a un año de su entrega observados en este estudio.

\section{Conclusión}

El envejecimiento de las poblaciones es una realidad que viene de la mano con cambios epidemiológicos, como el aumento progresivo de la prevalencia de hipoacusia. Desde hace una década, Chile cuenta con un programa ejemplar a nivel mundial, como es la garantía GES de "Hipoacusia bilateral en personas de 65 años y más que requieren uso de audífono". En este sentido, la adherencia terapéutica al uso del audífono es una preocupación constante y que debe ser evaluada de la mejor manera posible. Los resultados del presente análisis muestran de manera consistente que la adherencia al uso del audífono se encuentra en torno al $60 \%$ o más en Chile, llegando incluso a cifras alrededor de $73 \%-87 \%$, lo que representa una cifra muy positiva respecto a la efectividad de la política pública y permite plantear la necesidad de mantener y mejorar el beneficio a futuro.

\section{Bibliografía}

1. World Population Prospects: The 2012 Revision. United Nations Secretariat, 2014. (Disponible en http://esa.un.org/wpp/).

2. CEPAL. Observatorio Demográfico, 2013. Santiago de Chile.

3. Lee FS, Matthews LJ, Dubno JR, Mills JH. Longitudinal study of pure-tone thresholds in older persons. Ear Hear. 2005;26:1-11.

4. Yueh B, Shapiro N, MacLean CH, Shekelle PG. Screening and management of adult hearing loss in primary care: scientific review. JAMA. 2003;289:197685.

5. Walling AD, Dickson GM. Hearing loss in older adults. Am Fam Physician. 2012;85:1150-6.

6. World Health Organization. Millions of people in the world have hearing loss that can be treated or prevented. 2011 (Disponible en http://www.who.int/ pbd/deafness/news/Millionslivewithhearingloss.pdf).

7. Organización Panamericana de Salud / Organización Mundial de Salud. Guía Clínica para la Atención Primaria de las Personas Adultas Mayores, 2003.

8. Ministerio de Salud, Chile. Encuesta Nacional de Salud 2003.

9. Ministerio de Salud, Chile. Encuesta Nacional de Salud 2009.

10. Plomp R. Auditory handicap of hearing impairment and the limited benefit of hearing aids. J Acoust Soc Am. 1978;63:533-49.

11. Nabelek AK, Robinson PK. Monaural and binaural speech perception in reverberation for listeners of various ages. J Acoust Soc Am. 1982;71:1242-8.

12. Chia EM, Wang JJ, Rochtchina E, Cumming RR, Newall P, Mitchell P. Hearing impairment and health-related quality of life: the Blue Mountains Hearing Study. Ear Hear 2007;28:187-95.

13. Stark P, Hickson L. Outcomes of hearing aid fitting for older people with hearing impairment and their significant others. Int J Audiol. 2004;43:390-8.

14. Lin FR, Yaffe K, Xia J, et al. Hearing loss and 
cognitive decline in older adults. JAMA Intern Med 2013;173:293-9.

15. Ministerio de Salud, Chile. Guía Clínica "Hipoacusia bilateral en personas de 65 años y más que requieren uso de audífono", $1^{\text {a }}$ edición, junio de 2007.

16. Yueh B, Shapiro N, MacLean CH, Shekelle PG. Screening and management of adult hearing loss in primary care: scientific review. JAMA. 2003;289(15):1976-85.

17. Gates GA, Cobb JL, Linn RT, Rees T, Wolf PA, D'Agostino RB. Central auditory dysfunction, cognitive dysfunction, and dementia in older people. Arch Otolaryngol Head Neck Surg. 1996;122:161-7.

18. Gopinath B, Wang JJ, Schneider J, et al. Depressive symptoms in older adults with hearing impairments: the Blue Mountains Study. J Am Geriatr Soc. 2009;57:1306-8.

19. Karpa MJ, Gopinath B, Beath K, et al. Associations between hearing impairment and mortality risk in older persons: the Blue Mountains Hearing Study. Ann Epidemiol. 2010;20:452-9.

20. Chang HP. Presbycusis among older Chinese people in Taipei, Taiwan: a Community-based study. Int J Audiol. 2007;46(12):738-45.

21. Dunlop RJ, Dennis KC, Gonzenbach SA, Abrams HB, Berardino JT, Styer SA, Hall A. Support personnel in VA audiology. Audiology Today. 2006;18(1):24-5.

22. Joore MA, Potjewijd J, Timmerman AA, Anteunis LJ. Response shift in the measurement of quality of life in hearing impaired adults after hearing aid fitting. Qual Life Res 2002;11:299-307.

23. Mener DJ, Betz J, Genther DJ, Chen D, Lin FR. Hearing loss and depression in older adults. J Am Geriatr Soc 2013;61:1627-9.

24. Kochkin S. Hearing loss treatment. Better Hearing Institute. 2012. (Disponible en http://www. betterhearing.org/hearing_loss_treatment/index. (fm).

25. Solheim J, Kværner KJ, Falkenberg E-S. Daily life consequences of hearing loss in the elderly. Disability and Rehabilitation. 2011;33:2179-85.

26. McArdle R, Chisolm TH, Abrams HB, Wilson RH, Doyle PJ. The WHO-DAS II: measuring outcomes of hearing aid intervention for adults. Trends Amplif 2005;9:127-43.

27. Chisolm TH, Johnson CE, Danhauer JL, et al. A systematic review of health-related quality of life and hearing aids: final report of the American Academy of Audiology Task Force On the Health-Related Quality of Life Benefits of Amplification in Adults. J Am Acad Audiol 2007;18:151-83.

28. Popelka MM, Cruickshanks KJ, Wiley TL, Tweed TS, Klein BE, Klein R. Low prevalence of hearing aid use among older adults with hearing loss: the
Epidemiology of Hearing Loss Study. J Am Geriatr Soc. 1998;46:1075-8.

29. Kochkin SMV. Consumer satisfaction revisited. Hearing Journal. 2000;53:38-55.

30. Pacala JT, Yueh B. Hearing deficits in the older patient: "I didn't notice anything". JAMA. 2012;307:1185-94.

31. Infante A, Paraje G. Reforma de Salud: Garantías Exigibles como Derecho Ciudadano. In: Larrañaga O, Contreras D, eds. Las Nuevas Políticas de Protección Social en Chile. Santiago de Chile: Uqbar Editores; 2010;77-118.

32. Tamblay N, Villalobos I, Pastene A, Rahal M. Impacto social del uso de audífonos en adultos mayores. Rev Otorrinolaringol Cir Cabeza Cuello. 2008;68:21-6.

33. León A, Ediap R, Carvallo R. Adherencia al uso de audífonos en adultos mayores del Servicio de Salud Aconcagua. Rev Otorrinolaringol Cir Cabeza Cuello. 2010;70:37-42.

34. Maul X, Rivera C, Aracena K, Slater F, Breinbauer H. Adherencia y desempeño auditivo en uso de audífonos en pacientes adultos hipoacúsicos atendidos en la Red de Salud UC. Rev Otorrinolaringol Cir Cabeza Cuello. 2011;71:225-230.

35. Farfán C, Aguilera E, Lecaros R, Riquelme K, Valenzuela M, Manque P. No adherencia al uso de audífonos en adultos mayores de 65 años. Programa GES, Hospital Carlos Van Buren, 2014. Rev Chil Salud Pública. 2015;19(3):251-260.

36. Goulios H, Patuzzi RB. Audiology education and practice from an international perspective. Int $J$ Audiol. 2008;47:647-64.

37. Guerra-Zúñiga M, Cardemil-Morales F, AlbertzArévalo N, Rahal-Espejo M. Explicaciones al no uso de audífonos en un grupo de adultos mayores de Santiago de Chile. Un estudio cualitativo. Acta Otorrinolaringol Esp. 2014;65(1):8-14.

38. Sandín-Vázquez M, Sarría-Santamera A. Evaluación de impacto en salud: valorando la efectividad de las políticas en la salud de las poblaciones. Rev Esp Salud Pública. 2008;82:261-272.

39. Petticrew M, Whitehead M, Macintyre SJ, Graham H, Egan M. Evidence for public health policy on inequalities: 1: the reality according to policymakers. J Epidemiol Community Health. 2004 Oct;58(10):811-6.

40. Whitehead M, Petticrew M, Graham H, Macintyre SJ, Bambra C, Egan M. Evidence for public health policy on inequalities: 2: assembling the evidence jigsaw. $J$ Epidemiol Community Health. 2004;58(10):817-21.

41. Cardemil F, Muñoz D, Fuentes E. Hipoacusia asociada al envejecimiento en Chile: ¿En qué aspectos se podría avanzar? Rev Otorrinolaringol Cir Cabeza Cuello. 2016;76:127-135. 